\title{
Photon Correlation Spectroscopy and SAXS Study of Cylindrical to Spherical Transition in the AOT Microemulsion by Changing Solvent
}

\author{
Khadijeh Nikjoo $^{1}$, Mousa Aliahmad ${ }^{1^{*}}$, Soheil Sharifi $^{{ }^{*}}$, Mohsen Sargazi $^{2}$ \\ ${ }^{1}$ Department of Physics, University of Sistan and Baluchestan, Zahedan, Iran; ${ }^{2}$ Faculty of Science, Payame Noor University, Zahedan, \\ Iran. \\ Email: ${ }^{*}$ aliahmad@phys.usb.ac.ir, ${ }^{*}$ soheil.sharifi@gmail.com
}

Received January $26^{\text {th }}, 2012$; revised February $20^{\text {th }}, 2012$; accepted March $6^{\text {th }}, 2012$

\begin{abstract}
We study dynamic and structure of nano-droplets of AOT/water/oil microemulsion with different oils at water/AOT molar ratio of 6.7. Photon correlation spectroscopy is used to study collective diffusion coefficient of nano-droplets at AOT microemulsion with decane and cyclohexane. The collective diffusion coefficient of nanodroples is increasing with change of oil from decane to cyclohexane. The structure of AOT microemulsion with decane and cyclohexane is founded with SAXS. Our results show, nano-droplets of AOT microemulsion with decane at $\mathrm{X}=6.7$ have cylindrical structure and AOT microemulsion with cyclohexane at $\mathrm{X}=6.7$ have spherical structure.
\end{abstract}

Keywords: Nano-Size; Droplet; Microemulsions; Photon Correlation Spectroscopy; SAXS; Diffusion

\section{Introduction}

One of the interesting topics in the soft matter physics is study of dynamic of nano-droplets inside of microemulsion. In general, shape and size of nanodroplets are depends to the solutions of microemulsion, for example using the different oil can change the structure of nano-droplets. Microemulsions are thermodynamically stable mixtures of water, oil and surfactant with nano-meter size of droplets and oil is usually a hydrocarbon or a solution of a solute in a hydrocarbon [1-4]. The microemulsions have several application, they can be used to deliver drugs to the patients via several routes $[5,6]$. The anionic surfactant Aerosol OT (sodium bis-(2-ethylhexyl) sulfosuccinate, AOT) together with water and oil readily forms ternary microemulsions $[7,8]$. The AOT microemulsion at $\mathrm{X}=$ 6.7 show a single water-in-oil (L2) phase at the room temperature. The composition of each system is determined by the molar ratio $\mathrm{X}$ of water to surfactant molecules, $\mathrm{X}=$ $\left[\mathrm{H}_{2} \mathrm{O}\right] /[\mathrm{AOT}]$ and the droplet mass fraction $\mathrm{mf}=$ $\left(\mathrm{mAOT}+\mathrm{mH}_{2} \mathrm{O}\right) /\left(\mathrm{mAOT}+\mathrm{mH}_{2} \mathrm{O}+\mathrm{mDec}\right)$ Which varies by the respective mass of the components water $\left(\mathrm{mH}_{2} \mathrm{O}\right)$, decane $(\mathrm{mDec})$, and AOT.

Brownian motion is a subject of renewed interest since the development of photon correlation spectroscopy (PCS) in the last decade [9]. The dynamic properties of microemulsions and colloidal systems are studied by measuring

Corresponding authors. the relaxation of concentration fluctuations [9]. The study of the collective diffusion coefficient of microemulsion does give information on the interaction between droplets, [10]. A study shown, for $\mathrm{AOT} / \mathrm{H}_{2} \mathrm{O} /$ Decane microemulsion, at the low droplet mass fractions $(0.01<\mathrm{mf}<$ 0.1 transition from dilute to semi-dilute regime) and molar ratio of 40 the collective diffusion coefficient has a linear function of the mass fraction [10-12]. In the present work, we studied the behavior of the collective diffusion coefficient at the AOT microemulsion with decane and cyclohexane at molar ratio of 6.7 by photon correlation spectroscopy (PCS) and SAXS techniques.

\section{Experimental}

\subsection{Materials}

The microemulsions were prepared by mixing of surfactants AOT, $\mathrm{H}_{2} \mathrm{O}$, and oil (Decane and Cyclohexane) waiting for several minutes until the samples were single phase and optically clear. Sodium-2-diethylhexyl sulfosuccinate, or AOT 99\% (an Alfa product) and Decane 99\% (Aldrich) and Cyclohexane was obtained from Sigma-Aldrich. The composition of the AOT/ $\mathrm{H}_{2} \mathrm{O} / \mathrm{Oil}$ microemulsion is given by the two parameters $\mathrm{X}$ and $\mathrm{mf}$.

The experimental solutions were prepared by weighting appropriate amounts of AOT dissolving in decane or Cyclohexane and adding water into the solution. Finally, 
all samples were filtered by using 0.2-pm Teflon filters (Gelman).

\subsection{Methods}

\subsubsection{Small Angel $X$-Ray Scattering}

The measurements were performed on the modified NanoSTAR SAXS at the University of Aarhus. The instrument is a modified version of commercially available SAXS equipment (Bruker AXS). It is optimized with respect to flux and background and therefore ideally suited for solution scattering. The instrument uses a rotating anode X-ray source and is equipped with multilayer parabolic Gobel mirrors. The configuration of the instrument in the high-resolution configuration provides a range of scattering vector moduli $\mathrm{q}$ from 0.004 to 0.22 $\AA-1$ with a flux of 106 photons per second. The sample to detector distance was $106.70 \mathrm{~cm}$. The high-resolution version uses small pinholes $(0.5 \mathrm{~mm} \mathrm{~L}$ of source pinhole, $0.15 \mathrm{~mm} \mathrm{~L}$ of defining pinhole, and $0.5 \mathrm{~mm} \mathrm{~L}$ of the antiscatter pinhole in front of the sample) and a small beamstop $(2.0 \mathrm{~mm} \mathrm{~L})$. It can thus reach smaller values of the scattering vector. The sample was held in a $1 \mathrm{~mm}$ glass capillary at $\mathrm{T}=293.15 \mathrm{~K}$.

\subsubsection{Photon Correlation Spectroscopy}

Dynamic light scattering measurements were performed on filtered samples using Malvern photon correlation spectroscopy instrument at Ferdowsi University of Mashhad. The light source is a HeNe-laser, operating at a wavelength of $632.8 \mathrm{~nm}$, with vertically polarized light. The beam was focused on the sample cell (10-mm glass tubes) through a temperature-controlled cylindrical quartz container (with two plane-parallel windows), which is filled with a refractive index matching liquid (toluene). The temperature was kept at $293.15 \mathrm{~K}$ with an accuracy of $\pm 0.1 \mathrm{~K}$ during the experiments. The scattering angle $\theta$ was varied from $50^{\circ}$ to $110^{\circ}$ with $10^{\circ}$ increments, corresponding to a range of scattering wave vectors $(q)$ between $1.2 \times 107$ and $2.3 \times 107 \mathrm{~m}^{-1}$.

$$
q=\frac{4 \pi n}{\lambda} \sin \frac{\theta}{2}
$$

where $n$ is the refractive index of the solvent, $\lambda$ is the wavelength of the laser or x-ray, and $\theta$ is the scattering angle.

\section{Results and Discussion}

\subsection{Photon Correlation Spectroscopy}

Microemulsions were formulated by mixing AOT with water and n-Decane or cyclohexane at the fixed molar ratio of water to AOT $(\mathrm{X}=6.7)$ at the different mass fraction $(0.01<\mathrm{mf}<0.07$ dilute regime $)$. Dynamic be- havior of the AOT $/ \mathrm{H}_{2} \mathrm{O} /$ Decane or $\mathrm{AOT} / \mathrm{H}_{2} \mathrm{O} /$ Cyclohexane microemulsion was probed with photon correlation spectroscopy methods. The correlation function showed a single exponential decay at all concentrations, Figure 1 and Figure 2. In the photon correlation spectroscopy experiments, the normalized intensity time correlation function $g_{2}(q, t)$ was measured $[13,14]$,

$$
g_{2}(q, t)=\frac{\langle I(q, 0) I(q, t)\rangle}{\langle I(q, 0)\rangle^{2}}
$$

where $I(q, t)$ is the scattered intensity at a given $\mathrm{q}$ and time $t$. The $g_{2}(q, t)$ function is related to the normalized electrical field correlation function $g_{1}(q, t)$ by the Siegert relation assuming that the system is an ergodic media

$$
g_{2}(q, t)=1+B\left|g_{1}(q, t)\right|^{2}
$$

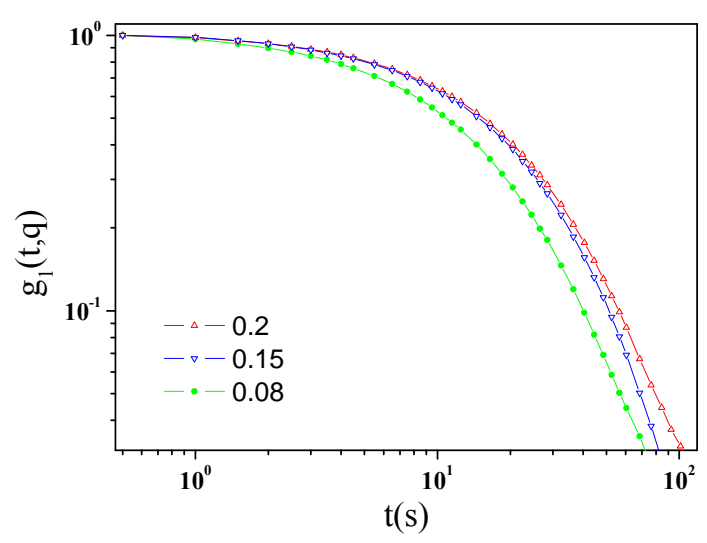

Figure 1. Correlation function as function of delay time of AOT $/ \mathrm{H}_{2} \mathrm{O} /$ Decane microemulsion at $\mathrm{X}=6.7$ with different mass fraction. All data of the correlation functions as function of delay time show a single stretched exponential function, at 293.15 K.

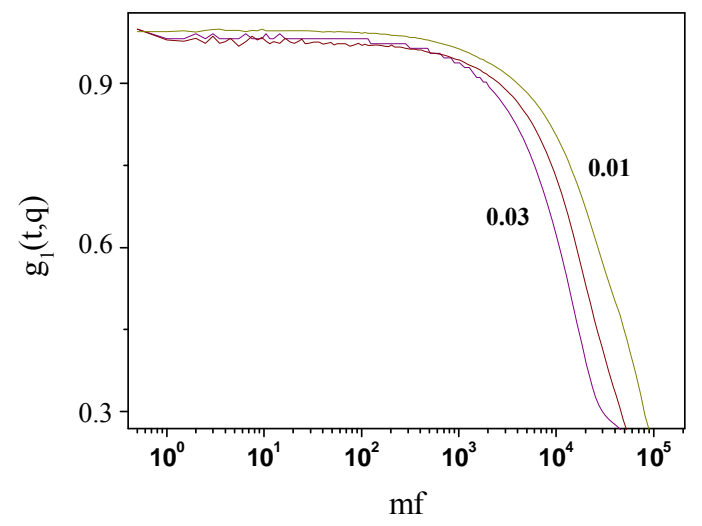

Figure 2. Correlation function as function of delay time of AOT $/ \mathrm{H}_{2} \mathrm{O} / \mathrm{Cyclohexane}$ microemulsion at $\mathrm{X}=6.7$ with different mass fraction. All data of the correlation functions as function of delay time show a single stretched exponential function, at 293.15 K. 
where $B$ is the coherence factor of the equipment. All the correlation functions in this work were fitted by a single stretched exponential function [15].

$$
g_{1}(t)=\exp \left[-\left(\frac{t}{\tau}\right)^{\beta}\right]
$$

The stretched exponential function describes the decay processes that have a distribution of relaxation times $(\tau)$. The parameter $\beta(0 \leq \beta \leq 1)$ measures the width of the distribution function. The results of the correlation function as function of delay time for AOT/ $\mathrm{H}_{2} \mathrm{O} /$ Decane at $\mathrm{X}=$ 6.7 at different mass fraction shown in the Figure 1. The correlation function as function of delay time for AOT/ $\mathrm{H}_{2} \mathrm{O} /$ Cyclohexane at $\mathrm{X}=6.7$ is presented in the Figure 2.

The mean value of the relaxation time is given by

$$
\langle\tau\rangle \equiv \int_{0}^{\infty} \exp \left[-\left(\frac{t}{\tau}\right)^{\beta}\right] \mathrm{d} t=\frac{\tau}{\beta} \Gamma\left(\frac{1}{\beta}\right)
$$

where $\Gamma$ is the gamma function. Where the collective diffusion coefficient is calculated from Dc $=1 /\left(q_{2}<\tau>\right)$. All the correlation functions in this work were fitted by a single stretched exponential function, Equation (4). The collective diffusion coefficient Dc were extracted as the function of the droplet mass fraction and illustrated in Figure 3. The collective diffusion coefficient measurements were carried out on dilution series of microemulsion samples at fixed temperature $293.15 \mathrm{~K}$ and molar ratio 6.7. In the Figure 3, the collective diffusion coefficient show a liner behavior with negative slop between $0.01<$ $\mathrm{mf}<0.07$. The light scattering experiments revealed that the collective diffusion coefficient of the system decreases with increasing droplet mass fraction, which indicates the attractive interactions between droplets.

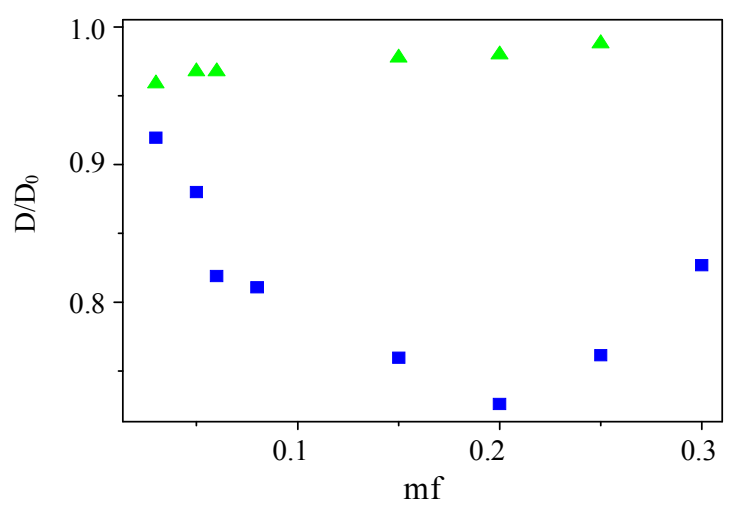

Figure 3. the collective diffusion coefficient as a function of mass fraction for $\mathrm{AOT} / \mathrm{H}_{2} \mathrm{O} /$ Decane microemulsion at $\mathrm{X}=$ 6.7 with different mass fraction (cubic) and $\mathrm{AOT} / \mathrm{H}_{2} \mathrm{O}$ / Cyclohexane microemulsion at $\mathrm{X}=6.7$ with different mass fraction (Up triangle) at the temperature 293.15K.

\subsection{Small Angle $X$-Ray Scattering}

In this work, SAXS was used for the determination of the structure of $\mathrm{AOT} / \mathrm{H}_{2} \mathrm{O} /$ Decane and $\mathrm{AOT} / \mathrm{H}_{2} \mathrm{O} / \mathrm{Cyclo}-$ hexane microemulsion at $293.15 \mathrm{~K}$. The scattered intensity as a function of $\mathrm{q}$ for $\mathrm{AOT} / \mathrm{H}_{2} \mathrm{O} /$ Decane and AOT/ $\mathrm{H}_{2} \mathrm{O} /$ Cyclohexane with molar ration 6.7 and mass fractions 0.1 were collected for these experiments is presented in the Figure 4, which the lines are fits to a power law, $I(q) \approx q-1$, with properties of a cylindrical object. At small $q$, the Guinier's law, $I(q) \approx \exp \left(-(q \xi)^{2 / 3}\right)$, is used for analyzing the data, where $\xi$ is a correlation length.

Results show that the correlation length $(\xi)$ is changing from $45 \AA$ to $23 \AA$ and cylindrical to spherical transition with changing oil from decane to cyclohexane. We know that spherical droplet with lower size can diffusion better that the cylindrical shape with bigger size. So, the change of size and shape can describe the different between the collective diffusion coefficient at $\mathrm{AOT} / \mathrm{H}_{2} \mathrm{O} /$ Decane and AOT $/ \mathrm{H}_{2} \mathrm{O} / \mathrm{Cyclohexane}$ microemulsion. For analyzing the data, we applied a model for a mixture of core-shell spheres with an added depletion attraction due to dissolved non-adsorbing polymer. The scattering intensity as function of the scattering vector $I(q)$ of spherical, monodisperse particles can be describe with a form factor component $F(q)$, which is proportional to the scattering of a single particle, and a structure factor $S(q)$, which describes the interaction effect:

$$
I(q)=c F^{2}(q) S(q)
$$

$c$ being a prefactor, which contains the number density of scattering particles. For the general case of $n$ shells around a spherical droplet core the form factor reads.

$$
F(q)=4 \pi \sum_{i=0}^{n} \Delta \rho_{i}\left(\frac{\sin \left(q R_{i}\right)-q R_{i} \cos \left(q R_{i}\right)}{q^{3}}\right)
$$

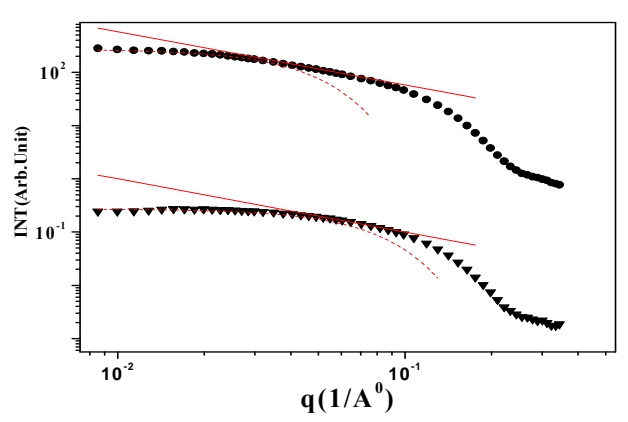

Figure 4. SAXS intensity $I(q)$ of a $\mathrm{AOT} / \mathrm{H}_{2} \mathrm{O} /$ Decane and $\mathrm{AOT} / \mathrm{H}_{2} \mathrm{O} / \mathrm{Cyclohexane}$ microemulsion at $\mathrm{X}=6.7$. The red line is the $I(q) \approx q-1$ that shown cylinder behavior of the SAXS experiments and dot line at low $q$ is the fit of Guinier's law, $I(q) \approx \exp \left(-(q \xi)^{2 / 3}\right)$. line is the $I(q) \approx q-1$ that shown cylinder behavior of the SAXS experiments and dot line at low $q$ is the fit of Guinier's law, $I(q) \approx \exp \left(-(q \xi)^{2 / 3}\right)$. 
where $R_{i}$ is the radius of the ith shell or, respectively, the core $R_{0}$ and $\Delta \rho_{i}$ is the electron density contrast between the shells $i$ and $i+1$ with $\rho n+1$ and $\rho_{0}$ being the electron density of the solvent and the core, respectively. So, for a simple core-shell micelle $n=1$. The structure factor is the Fourier transform of the pair correlation function $g(r)$.

$$
S(q)=1+4 \pi n \int_{0}^{\infty}(g(r)-1) \cdot r^{2} \frac{\sin (q r)}{q r} \mathrm{~d} r
$$

The pair correlation function gives the probability to find another particle at a distance $r$ from the center of a given particle, relative to the probability to find a particle at this distance in an ideal gas. It is closely related to the total correlation function $h(r)=g(r)-1$ and it can by means of the Ornstein-Zernike equation [16,17]. The results of the fit of SAXS experiment with hard sphere model and hard sphere model with core radius 25 and core-shell radius 30 with polydispersity 0.19 are presented in the Figure 5.

\section{Conclusion}

In this work, we found the collective diffusion coefficient of nano-droplets at $\mathrm{AOT} / \mathrm{H}_{2} \mathrm{O} /$ cyclohexane is higher than $\mathrm{AOT} / \mathrm{H}_{2} \mathrm{O} /$ Decane microemulsion at same molar ratio 6.7. Small-angle $\mathrm{X}$-ray scattering measurements revealed that shape of nano-droplets at $\mathrm{AOT} / \mathrm{H}_{2} \mathrm{O} /$ decane microemulsions change from cylindrical to spherical with change oil to cyclohexane. The increase of collective diffusion coefficient of nano-droplets at $\mathrm{AOT} / \mathrm{H}_{2} \mathrm{O} /$ cyclohexane instead of AOT $/ \mathrm{H}_{2} \mathrm{O} /$ Decane can be describe with spherical shape of $\mathrm{AOT} / \mathrm{H}_{2} \mathrm{O} /$ cyclohexane instead of cylindrical shape of $\mathrm{AOT} / \mathrm{H}_{2} \mathrm{O} /$ Decane.

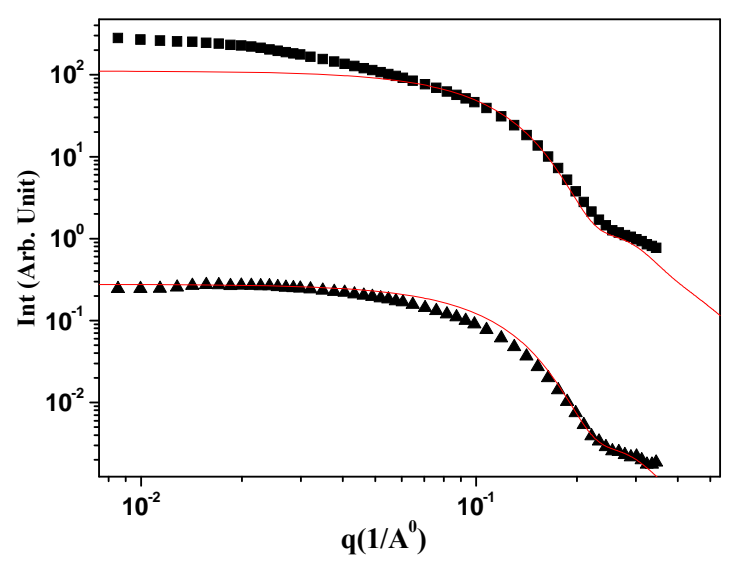

Figure 5. The SAXS intensity $I(q)$ of a $\mathrm{AOT} / \mathrm{H}_{2} \mathrm{O} / \mathrm{Decane}$ and $\mathrm{AOT} / \mathrm{H}_{2} \mathrm{O} / \mathrm{Cyclohexane}$ microemulsion at $\mathrm{X}=6.7$. The red line is the analyzing data with hard sphere model with core radius 25 and core-shell radius 30 with polydispersity 0.19.

\section{Acknowledgements}

This work was supported by the University of Sistan and Baluchestan.

\section{REFERENCES}

[1] S. Sharifi and A. Alavi, "Dynamic Light Scattering Study of Microemulsion," Proceedings of the SPIE, Vol. 8001, 2011, pp. 80012R-1-80012R-8.

[2] S. Sharifi and M. Amirkhani, "Light Scattering Study of Mixture of Polyethylene Glycol with C12E5 Microemulsion," Soft Nanoscience Letters, Vol. 1, No. 3, 2011, pp. 76-80. doi:10.4236/snl.2011.13014

[3] S. Sharifi, P. Kudla, C. L. P. Oliveira, J. S. Pedersen and J. Bergenholtz, "Variations in Structure Explain the Viscometric Behavior of AOT Microemulsions at Low Water/AOT Molar Ratios," Zeitschrift für Physikalische Chemie, Vol. 226, 2012, pp. 1-18. doi:10.1524/zpch.2012.0173

[4] S. Sharifi, M. Amirkhani, J. M. Asla, M. R. Mohammadi and O. Marti, "Light Scattering and SAXS Study of AOT Microemulsion at Low Size Droplet," Soft Nanoscience Letters, Vol. 2, No. 1, 2012, pp. 8-12. doi:10.4236/snl.2012.21002

[5] L. K. Pershing, L. D. Lambert and K. Knutson, "Mechanism of Ethanol-Enhanced Estradiol Permeation across Human Skin in Vivo," Pharmaceutical Research, Vol. 7, 1995, pp. 170-175.

[6] P. Liu, T. Kurihara-Bergstrom and W. R. Good, "Variation of Human Skin Permeation in Vitro: Ionic vs Neutral Compounds," Pharmaceutical Research, Vol. 8, No. 7, 1992, pp. 938-944. doi:10.1023/A:1015876117627

[7] E. V. Álvarez, J. Carballido-Landeira, J. Guiu-Souto, P. Taboada and A. P. Muñuzuri, "Communication: Spectroscopic Phase and Lineshapes in High-Resolution Broadband Sum Frequency Vibrational Spectroscopy: Resolving Interfacial Inhomogeneities of "Identical" Molecular Groups," Journal of Chemical Physics, Vol. 135, No. 24, 2011, p. 094512. doi:10.1063/1.3675629

[8] J. Carballido-Landeira, P. Taboada and A. P. Muñuzuri, "Nanoscale Changes Induce Microscale Effects in Turing Patterns," Physical Chemistry Chemical Physics, Vol. 13, No. 10, 2011, pp. 4596-4599. doi:10.1039/c0cp02362k

[9] W. Brown, "Dynamic Light Scattering: The Method and Some Applications," Clarendon, Oxford, 1993.

[10] T. Blochowicz, C. Gögelein, T. Spehr, M. Müller and B. Stühn, "Polymer-Induced Transient Networks in Waterin-Oil Microemulsions Studied by Small-Angle x-Ray and Dynamic Light Scattering," Physical Review E, Vol. 76, No. 4, 2007, pp. 041505-041513. doi:10.1103/PhysRevE.76.041505

[11] J. Bergenholtz, A. Romagnoli and N. J. Wagner, "Viscosity, Microstructure, and Interparticle Potential of AOT/ $\mathrm{H}_{2} \mathrm{O}$ /n-Decane Inverse Microemulsions," Langmuir, Vol. 11, No. 5, 1995, pp. 1559-1570. doi:10.1021/la00005a025

[12] U. Batra, W. B. Russel and J. S. Huang, "Viscosity 
Anomaly and Charge Fluctuations in Dilute AOT Microemulsions with $\mathrm{X}<20$," Langmuir, Vol. 15, No. 11, 1999, pp. 3718-3725. doi:10.1021/1a9812727

[13] H. Yang, G. Zheng, S.-G. Dai, R.-J. Zhang and P.-A. Mu, "Dynamic Light Back-Scattering with Polarization Gating and Fourier Spatial Filter for Particle Sizing in Concentrated Suspension," Optica Applicata, Vol. 40, No. 4, 2010, pp. 819-826.

[14] I. Block and F. Scheffold, "Modulated 3D Cross-Correlation Light Scattering: Improving Turbid Sample Characterization," Review of Scientific Instruments, Vol. 81, No. 12, 2010, p. 123107. doi:10.1063/1.3518961

[15] R. Bandyopadhyay, P. H. Mohan and Y. M. Joshi, "Stress Relaxation in Aging Soft Colloidal Glasses," Soft Matter,
Vol. 6, No. 7, 2010, pp. 1462-1466.

doi:10.1039/b916342e

[16] M. Nayeri, M. Zackrisson and J. Bergenholtz, "Scattering Functions of Core-Shell-Structured Hard Spheres with Schulz-Distributed Radii," Journal of Physical Chemistry $B$, Vol. 113, No. 24, 2009, pp. 8296-8302. doi:10.1021/jp811482w

[17] M. Schwab and B. Stuhn, "Relaxation Phenomena and Development of Structure in a Physically Cross Linked Nonionic Microemulsion Studied by Photon Correlation Spectroscopy and Small Angle X-Ray Scattering," Journal of Chemical Physical, Vol. 112, No. 14, 2000, pp. 6461-6471. doi:10.1063/1.481207 